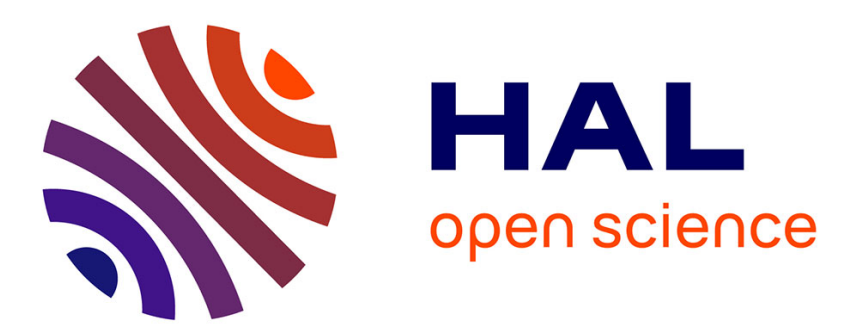

\title{
Un cadre téphrostratigraphique réactualisé pour la préhistoire tardiglaciaire et holocène de Limagne (Massif central, France)
}

Gérard Vernet, Jean-Paul Raynal

\section{- To cite this version:}

Gérard Vernet, Jean-Paul Raynal. Un cadre téphrostratigraphique réactualisé pour la préhistoire tardiglaciaire et holocène de Limagne (Massif central, France). Comptes rendus de l'Académie des sciences. Série IIa, Sciences de la terre et des planètes, 2000, 330, pp.399-405. halshs-00004134

\section{HAL Id: halshs-00004134 \\ https://shs.hal.science/halshs-00004134}

Submitted on 14 Jul 2005

HAL is a multi-disciplinary open access archive for the deposit and dissemination of scientific research documents, whether they are published or not. The documents may come from teaching and research institutions in France or abroad, or from public or private research centers.
L'archive ouverte pluridisciplinaire HAL, est destinée au dépôt et à la diffusion de documents scientifiques de niveau recherche, publiés ou non, émanant des établissements d'enseignement et de recherche français ou étrangers, des laboratoires publics ou privés. 


\title{
Un cadre téphrostratigraphique réactualisé pour la préhistoire
} tardiglaciaire et holocène de Limagne (Massif central, France). Tephrostratigraphy of Limagne revisited. Implications for Late Glacial and Holocene Prehistory.

\section{Gerard Vernet ${ }^{1}$ et Jean-Paul Raynal ${ }^{2}$}

\author{
'AFAN, UPRES-A 6042 et GDR 1122 CNRS, 7 rue du Mont Mouchet, 63320 Chadeleuf,France \\ ${ }^{2}$ Institut de Préhistoire et de Géologie du Quaternaire, UMR CNRS 5808, \\ Université de Bordeaux 1, Avenue des Facultés, F-33405 TALENCE et GDR 1122 CNRS. \\ jpraynal@wanadoo.fr
}

Abstract : From the Older Dryas to the Atlantic Period, ten pyroclastic formations originating from trachyandesitic and trachytic eruptions covered the plain of the Limagne d'Auvergne and have been preserved in various depositional contexts. They are useful isochronic markers for archaeologists examining their impact on prehistoric behaviours and settlements.

Key-words : Tephras, Late-Glacial, Holocene, Limagne, Massif Central, Prehistory

Resume : Dix recouvrements de tephras, suffisamment importants pour avoir ete préservés en stratigraphie dans des environnements sédimentaires divers, ont affecté la Limagne du Dryas ancien à l'Atlantique. Ils caractérisent des éruptions pour l'essentiel à magmas trachyandésitiques et trachytiques. Outre leur rôle incontestable de marqueurs isochroniques, ils incitent à reconsidérer l'impact du volcanisme de la Chaîne des Puys sur l'environnement des peuplements préhistoriques contemporains.

Mots-cles : tephras, Tardiglaciaire, Holocène, Limagne, Massif Central, préhistoire

Following preliminary research (Brousse et al.,1969 ; Baudry et Camus, 1972 ; Camus, 1975) and a detailed synthesis based on a pluridisciplinary approach (Vernet,1992), the tephostratigraphy of Limagne has been completed (Vernet et al., 1998) and detailed, identifying ten markers which are characterized by their facies, chemistry (in $\mathrm{SiO}_{2} / \mathrm{Na}_{2} \mathrm{O}+\mathrm{K}_{2} \mathrm{O}$ diagram) (Le Bas et al, 1985) (figure 
1), heavy mineral content and granulometry (Fischer, 1961). They have been directly TL dated or are associated with palaeosoils dated by radiocarbon, or occur within palynological sequences. From the oldest to the youngest, the identified tephras are:

1 - The Cellule Tephra. This is a coarse ash composed of trachyandesite, observed at Cellule within the Orange marsh and dated by palynology to the Ancient Dryas (Vernet and Paquereau, 1986, 1991 ; Vernet et al., 1990). The mineralogical association is dominated by a green clinopyroxene (94.5\%). It is strongly deformed by frost (figure 2), but one can estimate its thickness to be between 0.20 and $0.30 \mathrm{~m}$. The source volcano is probably the Puy de La Nugère, during one of the oldest eruptive phases.

2 - CF1a/ CF1b Tephric Complex (La Barre Street fallout/Les Roches fallout). About thirty observed sites distributed in the Limagne show that this is composed of a black basal level more or less developed (CF1a or La Barre Street fallout) followed by an upper grey level (CF1b or Les Roches fallout).

CF1a, composed of basaltic trachyandesite, presents a facies in centimetric regular black beds grinding the existing topography with a basaltic composition. Its extension is less important than in CF1b : the observed sites are confined to the Clermont Basin (La Barre Street, Montjuzet Street, Blanzat Street) as well as in Gerzat-Cebazat and Le Brezet areas.

CF $1 \mathrm{~b}$ is bedded, with more or less thick lamina, composed of trachyandesite with brown amphibole as a characteristic mineral. It is composed of material accumulated in channels where intersected stratification can appear.

The facies are present in several stratigraphies in the Clermont (La Barre Street, Montjuzet Street...), in the Grande Limagne, in the Morge area (Gimeaux) and in the Magdalenian prehistoric site of Enval. This covering has 
therefore been widespread in the Limagne and its average thickness is around $0.15 \mathrm{~m}$. A rapid calculation shows that $1500 \mathrm{~m} 3$ ashes per hectare have covered the Limagne around 12000 years BP. Its impact on the biotopes is evidenced by the palynology (D. Vivent, unpublished). This tephric complex is the result of an eruption beginning with a single magma phase that was trachyandesitic basaltic in nature (CF1a), then continued with a mix of trachyandesitic basaltic and trachyandesitic magmas, from which derive the presence of the two scorias facies in $\mathrm{CF} 1 \mathrm{~b}$. Only Puy de la Nugère has provided lavas where one observes all stages of two mix magmas during an eruptive phase with a compatible age (Gourgaud \& Camus, 1984). This tephric complex is dated by ${ }^{14} \mathrm{C}$ on the underlying paleosols (Ly 338/ AA-22073 = $11990 \pm 90$ and ARC $1697=11460 \pm 90$ ) and directly on organic fragments within the tephra (Gif TAN $91102=12010 \pm 150$ BP). The Les Roches Tephra has directly affected a Late Magdalenian living site, Abri Durif at Enval, $30 \mathrm{~km}$ to the southeast of its source (Vernet, 1992 ; Vernet \& Raynal, 1995) : the uppermost layer is composed of a thin, regular, well-sorted fall ( $2 \mathrm{~cm}$ thick) which adheres to the stone artefacts and faunal remains of the most recent Magdalenian occupation and thus seals the archaeological sequence.

3 - La Moutade Tephra is a black coarse ash with a basaltic trachyandesite composition seen at a number of locations in the western Limagne (Orange Marsh, Sardon valley and Villeneuve-les-Cerfs Marsh). The mineralogical association is clearly dominated by brown amphiboles (70 to 85\%). Puy de la Nugère is proposed as the source (Etlicher et al. , 1987 ; Juvigné et al., 1991). Direct dating has been obtained by thermoluminescence at $13700 \pm 1700$ (Cler TL 110), palynologic dating indicates the Alleröd and radiocarbon dating is $11360 \pm 130$ BP (Ly 3733) (Vernet and Paquereau, 1986, 1991 ; Vernet et al. , 1990). Its impact on the vegetation has been evidenced by the palynology on the Villeneuve-les-Cerfs site (Raynal et al., 1998). 
4 - The CF2 Tephra (or Descartes Street fallout). This occurs in centimetric light grey beds with numerous xenoclastes incorporated from the bedrock which lie in unconformity above CF1. It has a composition of basaltic trachyandesite and characteristic minerals are green clinopyroxene and brown amphibole. It is present in several places in the Clermont Basin, at Descartes Street, Sous-les-Vignes Street, Blanzat Street and Montjuzet Street. Dated directly by TL at $8700 \pm 900$ (Cler 114) (Raynal et al., 1989) and dated by palynology on another section near Clermont to the Pre-Boreal/Boreal boundary (Vernet et al., 1996 ; Raynal et al., 1998).

5 - The CF3 Tephra (or Montjuzet Street fallout). This has a composition of trachybasalt, with characteristic minerals being green clinopyroxene and olivine. It is present in all the studied stratigraphies of the Clermont Basin and in the Cebazat-Gerzat area. It presents a very characteritic facies : at the bottom a thin reddish level typical of a phreatomagmatic blast effect, then a mix of products characteristic of a mixed eruption (magmatic and phreatomagmatic), finally vesicular scorias characteristic of a strombolian plume fall (Vernet, 1992). These tephras are dated to the Boreal by palynology (Vernet et al., 1996). For now their origin remains uncertain.

6 - The CF4 Tephra (or Blanzat Street ancient fallout), is composed of basaltic trachyandesite with characteristic minerals being clinopyroxene and olivine. Its facies is an alternation of beds rich in vesiculated scorias and beds of finer granulometry rich in xenoclasts of the bedrock and cracked marl fragments (strongly phreatomagmatic characteristics). They are present at Blanzat Street and Montjuzet Street. Their chronological position is identical to that of CF3, at the limit of the Preboreal/Boreal (Vernet et al., 1996). The origin is uncertain but one can suppose that they come from the same 
structure as CF3.

7 - The CF5 Tephra (or Sous-les-Vignes Street fallout), is a coarse ash forming a centimetric lamina $(0.03 \mathrm{~m})$ discontinuous, cream to pastel rose, with a composition of rhyolite, with characteristic minerals being green clinopyroxene (55\%), zircon (16\%), brown amphibole (14\%) and apatite (10\%). This lamina is present in a few stratigraphies in the Clermont Basin (Sous-lesVignes Street) and in the Limagne near Gerzat (Daugas et Tixier, 1975 ; Juvigné et al., 1992 ; Raynal et al., 1998). It is dated palynologically at the Boreal/Atlantic limit (Vernet et al., 1996). The Kilian Crater has been proposed as source volcano (Vernet, 1992) after the lack of sphene which excludes the Puy Chopine (Bentor, 1955) and the stratigraphic position which excludes the Puy de Dôme, older after the TL dates (Faïn et al., 1986, 1991). But the Puy Vasset is a potential source (Michon, 1996). A very broad dispersal has been proposed for the trachytic tephras of the Chaîne des Puys (Puy de Vasset or Kilian Crater) of Boreal age (Juvigné, 1991, 1992).

8 - The Marsat Formation, eruptive and epi-eruptive born of a trachytic structure, formed of a mud-flow, torrential alluviums and ash levels, overlays two epipalaeolitic archaeological layers dicovered at les Pradelles in 1995 (Vernet et al, 2001). The geochemistry lies on the trachyte/rhyolite limit (figure 1) and the mineralogy of these products (presence of sphene) allows them to be sourced without ambiguity to the Puy Chopine, more than $11 \mathrm{~km}$ distant. Carbonized branches, discovered at the base of eruptive products of this volcano in proximal position (approximately $1 \mathrm{~km}$ from the protrusion), have given a radiocarbon age of $8464 \pm 70 \mathrm{BP}$, confirming previous results (Camus 1975, Raynal et al. 1981, Juvigné 1987). The importance and the nature of eruptive deposits from the Puy Chopine discovered at Marsat radically change the vision that one had until now of trachytic eruptions in the 
Chaîne des Puys : a syneruptive mud-flow hit the Limagne more than $11 \mathrm{~km}$ from its emission point around 8500 year BP.

9 - The CF6 Tephra (Blanzat Street recent fallout ), shows bedded levels with a fine granulometry where alternate dark and pale beds, rich in xenoclastes, composed of trachyandesite. Characteristic minerals are green and brown clinopyroxenes. It has only been observed in one place of the Clermont Basin (Blanzat Street) but could also have been present in the Gerzat area. The source volcano is doubtless the Puy de Pariou (Vernet, 1992), relating particularly to the main lava flow episode in the history of this volcano dated of $8180 \pm 810$ (Guérin, 1983).

10 - The CF7 Tephra is represented by centimetric angular fragments of trachyte noticed on several sections studied in the North-east, in the East and the South of Clermont-Ferrand, above the CF6 fallout and below an Ancient Neolithic archaeological layer $\left({ }^{14} \mathrm{C}\right.$ ETH-17946 $=5405 \pm 75$ BP $)$. The sole trachytic structure which lava possessing comparable contents is the Puy de Clierzou. The content analysis of radioelements ${ }^{232} \mathrm{Th}$ and ${ }^{214} \mathrm{U}$ confirms this origin (Miallier, pers. com). The morphology of trachytic fragments suggests that they come from the crust of a dome. They are therefore distal evidences of a primitive dome explosion of the Puy de Clierzou and form the more recent tephric covering in the Limagne.

11 - Other Tephras - Two tephras of unknown origin are still to be dated precisely : the Gimeaux Upper Tephra, with a trachyandesitic composition, deposited during the Late-Glacial sensu lato, and theRouzat Upper Tephra, composed of trachybasalt, probably deposited at the beginning of the Holocene (Vernet, 1992). 
12 - Conclusions - Late Glacial and Holocene volcanic activity of the Chaîne des Puys had a severe impact on the Limagne plain, especially during the Alleröd and the Boreal : there are no paleosoils between tephras CF2, CF3 and CF4, which are followed by several trachytic volcanic products leading up to the beginning of Neolithic times. Other more recent tephras have been observed on the Dômes plateau (Juvigné et al, 1986) but have not been recognized in the plain. Tephras have been preserved in various depositional contexts and have contributed to filling the Limagne hollows and greatly disturbing their regular evolution. A detailed tephrostratigraphic framework has thus been established. Archaeologists have now to determine precisely the consequences for prehistoric human groups.

A la suite de travaux initiaux (Brousse et al. 1969 ; Baudry et Camus 1972 ; Camus 1975) et de travaux pluridisciplinaires ayant conduit à une première synthèse très détaillée (Vernet, 1992), la téphrostratigraphie de Limagne a pu être complétée (Vernet et al., 1998) et précisée : on reconnaît aujourd'hui dix recouvrements téphriques principaux, caractérisés par leur faciès, leur chimisme (représenté dans un diagramme $\mathrm{SiO}_{2} / \mathrm{Na}_{2} \mathrm{O}+\mathrm{K}_{2} \mathrm{O}$ ) (Le Bas et al., 1985) (figure 1), leur cortège de minéraux denses et leur granulométrie (selon Fischer, 1961). Ils sont directement datés par thermoluminescence ou associés à des paléosols datés par le radiocarbone, ou encore inclus dans des séquences palynologiques. Nous les décrivons du plus ancien au plus récent.

1 - La retombée de Cellule est une cendre grossière de composition trachyandésitique, puissante de 0,20 à $0,30 \mathrm{~m}$, observée uniquement à Cellule dans le Marais d'Orange, où elle est fortement cryoturbée Le cortège minéralogique est dominé par un clinopyroxène vert (94,5\%). Elle a été située par la palynologie dans le Dryas ancien (Vernet et Paquereau, 1986, 1991 ; 
Vernet et al, 1990). II pourrait s'agir d'un recouvrement lié à une phase éruptive ancienne du Puy de la Nugère.

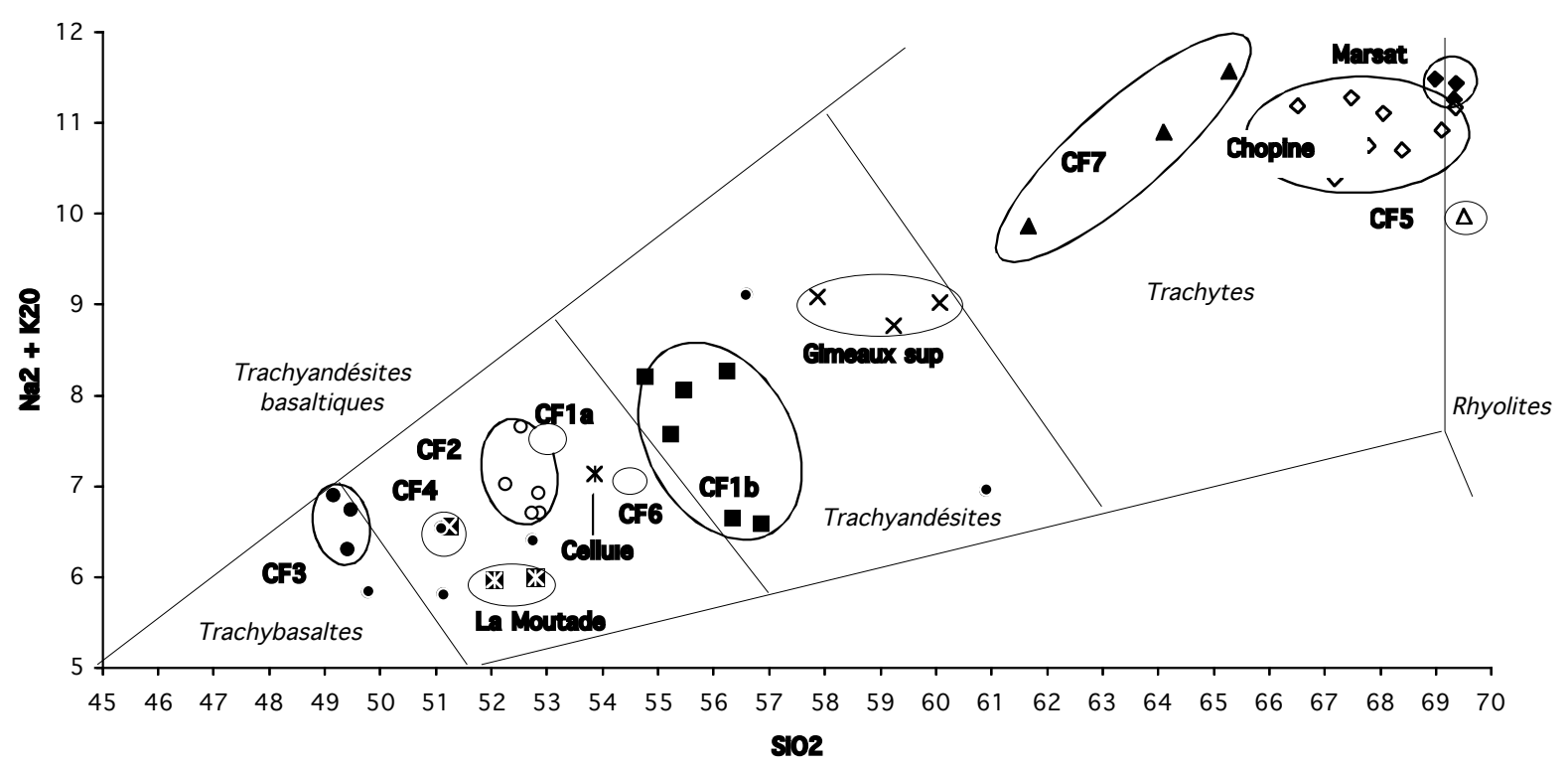

Figure 1 : Composition chimique des différents téphras reconnus dans un diagramme $\mathrm{SiO}_{2} / \mathrm{Na}_{2} \mathrm{O}+\mathrm{K}_{2} \mathrm{O}$ (Le Bas et al., 1985) Chemical composition of identified tephra in $\mathrm{SiO}_{2} / \mathrm{Na}_{2} \mathrm{O}+\mathrm{K}_{2} \mathrm{O}$ (weight\%) diagram (after Le Bas et al., 1985).

2 - Le Complexe téphrique CF1a/CF1b (retombée de la rue de la Barre/retombée des Roches).

Présent dans toute la Grande Limagne, il scelle le remplissage du site magdalénien d'Enval : c'est le premier téphra distal de la Chaîne des Puys retrouvé en contexte préhistorique. D'après une trentaine de sites d'observation répartis en Limagne, il est constitué d'un niveau de base noir plus ou moins développé (CF1a ou retombée de la rue de la Barre) puis d'un niveau sommital gris (CF1b ou retombée des Roches).

CF1a se présente en lits centimétriques réguliers de couleur noire moulant la topographie existante. Son extension est moins importante que celle de CF1b: les sites d'observations se cantonnent dans la cuvette de Clermont (rue de la Barre, rue de Montjuzet, rue de Blanzat) et dans les secteurs de 
Gerzat-Cébazat et du Brézet. Sa composition indique un magma trachyandésitique basaltique.

CF1b montre un litage net formé de lamines plus ou moins épaisses et forme des accumulations importantes en chenaux, parfois à stratifications entrecroisées. Sa composition entre dans le domaine des trachyandésites, avec comme minéral caractéristique l'amphibole brune. CF1b est présente dans plusieurs stratigraphies de la cuvette de Clermont (rue de la Barre, rue de Montjuzet...), en grande Limagne, dans le secteur de la Morge (Gimeaux) et enfin dans le site magdalénien d'Enval, où il adhère aux outils de pierre et aux ossements du niveau $1 \mathrm{a}$ et scelle la séquence archéologique, démontrant la pénécontemporanéité de l'occupation humaine et de l'éruption (Vernet et Raynal, 1995).

Ce recouvrement a donc été quasi général en Limagne et son épaisseur moyenne est de l'ordre de $0,15 \mathrm{~m}$. Un calcul rapide montre que $1500 \mathrm{~m}^{3}$ de cendres à l'hectare ont recouvert la Limagne vers 12000 ans BP. L'impact sur les biotopes est clairement mis en évidence par les analyses palynologiques réalisées de part et d'autre du niveau téphrique (Vivent, inédit).

Une des caractéristiques principales de ce recouvrement est la présence de deux faciès différents de scories définis au M.E.B. :

- des scories noires anguleuses à vésiculation peu à très peu développée (composition géochimique à la limite des domaines des trachyandésites basaltiques et des trachyandésites),

- des scories claires à l'aspect "moussu" ou "ponceux" à tendance fibreuse, à composition géochimique de trachyandésite.

L'analyse fine du complexe téphrique $\mathrm{CF} 1 \mathrm{a} / \mathrm{CF} 1 \mathrm{~b}$ permet une mise en corrélation avec un édifice de la Chaîne des Puys. L'éruption débute en effet par une phase à magma unique de nature trachyandésitique basaltique (CF1a), puis se poursuit avec un mélange de magmas trachyandésitique basaltique et trachyandésitique, d'où la présence des deux faciès de scories dans CF1b. Seul 
le Puy de la Nugère a fourni des laves où l'on observe tous les stades de mélange de deux magmas lors d'une phase éruptive d'âge compatible (Gourgaud et Camus, 1984). Le Puy de la Nugère est donc la source retenue pour ce recouvrement téphrique.

Ce complexe téphrique est daté par le ${ }^{14} \mathrm{C}$ sur des paléosols sous-jacents (Ly 338/AA-22073 = $11990 \pm 90$ et ARC $1697=11460 \pm 90)$ et directement sur des fragments organiques au sein du téphra (Gif TAN $91102=12010 \pm 150$ $B P)$.

3 - La retombée de la Moutade est une cendre grossière noire à composition de trachyandésite basaltique, reconnue en différents points de Limagne septentrionale (Marais d'Orange, vallée du Sardon et Marais de Villeneuve-lesCerfs). Le cortège minéralogique est nettement dominé par l'amphibole brune (70 à $85 \%$ ). Le puy de la Nugère est proposé comme volcan source source (Etlicher et al. , 1987 ; Juvigné et al., 1991). Une datation directe par thermoluminescence de $13700 \pm 1700$ (Cler TL 110) (Vernet et al., 1990), est confirmée par un calage palynologique dans l'Alleröd et une datation radiocarbone de $11360 \pm 130$ BP (Ly 3733) (Vernet et Paquereau, 1986, 1991; Vernet et al., 1990). Son impact sur la végétation a été mis en évidence par les analyses palynologiques réalisées par D. Vivent sur le site de Villeneuveles-Cerfs (Raynal et al., 1998).

4 - Le Téphra CF2 (ou retombée de la rue Descartes)

De composition de trachyandésite basaltique, avec des minéraux caractéristiques qui sont le clinopyroxène vert et l'amphibole brune, il se présente en lits centimétriques de couleur grise à xénoclastes du socle nombreux. On note la présence de niveaux de dépôts de pente carbonatés qui 
s'interstratifient dans la partie sommitale des cendres et attestent de processus de remaniement de versant. CF2 est présent en de nombreux points de la cuvette de Clermont, rue Descartes, rue Sous-les-Vignes, rue de Blanzat et rue de Montjuzet. Daté directement par TL à $8700 \pm 900$ (Cler 114) (Raynal et al, 1989), il est calé par la palynologie vers la transition Pré-Boréal/Boréal (Vernet et al. 1996, Raynal et al., 1998).

5 - LeTéphra CF3 (ou retombée de la rue de Montjuzet)

Avec une composition de trachybasalte et des minéraux caractéristiques qui sont le clinopyroxène vert et l'olivine, ces cendres moulent la topographie et sont présentes dans toutes les stratigraphies étudiées dans la cuvette de Clermont et dans le secteur de Cébazat-Gerzat. Elles présentent un faciès très particulier : à la base un mince niveau rougeâtre caractéristique d'un effet de "blast" phréatomagmatique, puis un mélange de produits caractéristiques d'une éruption mixte (magmatique et phréatomagmatique), enfin des scories vésiculées caractéristiques d'une retombée de panache strombolien (Vernet, 1992). Ces cendres sont situées par la palynologie dans le Boréal (Vernet et al., 1996), mais leur origine reste pour l'instant incertaine.

6 - Le Téphra CF4 (ou retombée ancienne de la rue de Blanzat)

Cette retombée présente une composition de trachyandésite basaltique. Ses minéraux caractéristiques sont le clinopyroxène et l'olivine. Son faciès est particulier : alternance de lits riches en scories vésiculées et de lits à granulométrie plus fine riches en xénoclastes du socle et fragments de marnes craquelées (caractère phréatomagmatique accusé). CF4 est présent rue Sousles-Vignes, rue de Blanzat et rue de Montjuzet. II se superpose directement à CF3 et la palynologie le situe également dans le Boréal (Vernet et al., 1996). 
L'origine est incertaine mais l'on peut supposer qu'il provient du même édifice que CF3.

7 - Le Téphra CF5 (ou retombée de la rue Sous-les-Vignes)

Cette lamine est présente dans une seule des stratigraphies de la cuvette de Clermont (rue Sous-les-Vignes) et également en Limagne dans le secteur de Gerzat. Dans cette dernière zone, plusieurs niveaux de téphras acides repérés en 1971 (Daugas et Tixier, 1975) ont été ultérieurement situés par la palynologie dans le Boréal (Raynal et al., 1998). Une très vaste dispersion a récemment été proposée pour des tephras trachytiques de la Chaîne des Puys (Vasset ou Kilian) d'âge Boréal (Juvigné, 1991, 1992 ; Juvigné et al., 1992).

Le téphra CF5 est une cendre grossière formant une lamine centimétrique $(0,03 \mathrm{~m})$, discontinue, de couleur crème à rose pastel, avec un granoclassement local. Elle présente une composition de rhyolite et les minéraux caractéristiques sont le clinopyroxène vert (55\%), le zircon (16\%), l'amphibole brune (14\%) et l'apatite (10\%). Elle est calée palynologiquement vers la transition Boréal/ début de l'Atlantique (Vernet et al., 1996). L'âge de cette retombée trachytique exclut de la rapporter au Puy-de-Dôme daté par TL (Faïn et al., 1986, 1991) et sa composition minéralogique caractérisée par l'absence de sphène élimine le Puy Chopine (Bentor, 1955). Le Cratère Kilian a été proposé comme volcan source (Vernet, 1992), mais la distinction entre ses produits et ceux du Puy Vasset reste délicate, l'éruption de ce dernier paraîssant en outre beaucoup plus importante (Michon, 1996).

\section{8 - La Formation de Marsat}


La Formation de Marsat a été identifiée en 1995 au lieu-dit Les Pradelles, où elle surmonte deux niveaux archéologiques épipaléolitiques. Elle est constituée d'une coulée boueuse à fragments anguleux de trachyte et empreintes végétales, d'alluvions torrentielles (composées exclusivement de blocs et galets de trachyte) et de cendres fines. La géochimie (à la limite des domaines trachyte/rhyolite) et la minéralogie de ces produits (présence de sphène) permettent de les attribuer sans ambiguïté au Puy Chopine, distant de plus de $11 \mathrm{~km}$ du site étudié. Des branches carbonisées, découvertes à la base des produits éruptifs de ce volcan en position proximale (environ $1 \mathrm{~km}$ de la protrusion), ont donné une date de $8465 \pm 70$ BP (ARC 1561), qui confirme les âges précédemment obtenus (Camus, 1975, Raynal et al. 1981, Juvigné 1987). L'importance et la nature des dépôts éruptifs du Puy Chopine découverts à Marsat changent de façon radicale la vision que les volcanologues avaient jusqu'à présent des éruptions trachytiques de la Chaîne des Puys : nul n'imaginait qu'une coulée boueuse synéruptive ait affecté la Limagne à plus de $11 \mathrm{~km}$ de son point d'émission vers 8500 BP.

9 - Le Téphra CF6 (ou retombée récente de la rue de Blanzat)

Cette retombée montre une composition de trachyandésite avec comme minéraux caractéristiques des clinopyroxènes verts et bruns. Son faciès est lité, de granulométrie fine, les lits plus clairs étant riches en xénoclastes de socle. Observée dans une seule coupe de la cuvette de Clermont (rue de Blanzat), elle est également présente dans le secteur de Gerzat. Elle n'est pas datée directement mais sa chimie la rapporte indiscutablement au Puy de Pariou et plus particulièrement à l'épisode d'émission de sa coulée principale datée de $8180 \pm 810$ (Guérin, 1983).

10 - Le Téphra CF7 (ou téphra du Clierzou) 
Depuis 1997, plusieurs coupes stratigraphiques au Nord-Est, à l'Est et au Sud de Clermont-Ferrand ont montré la présence d'un niveau limoneux contenant de nombreux fragments anguleux centimétriques de trachyte. Ce niveau surmonte la retombée CF6 et constitue la partie basale d'une couche contenant des vestiges du Néolithique ancien (épi-Cardial), daté de $5405 \pm 75$ BP (ETH17946). Les analyses géochimiques indiquent comme origine le Puy de Clierzou. L'analyse des radioéléments ${ }^{232} \mathrm{Th}$ et ${ }^{214} \mathrm{U}$ confirme cette origine (D. Miallier, com. pers.). La morphologie des fragments trachytiques suggère qu'ils proviennent de la croûte d'un dôme. CF7 représente donc les témoins distaux de l'explosion d'un dôme primitif du Clierzou et forment le recouvrement téphrique le plus récent de Limagne. II convient maintenant de vérifier si les dômes récents comme le Clierzou ne portent pas de recouvrements encore plus récents.

\section{1 - Autres téphras}

Deux retombées dont l'origine n'est pas identifiée sont encore mal datées : la retombée supérieure de Gimeaux, à composition trachyandésitique, observée dans les travertins hydrothermaux de la source de Gimeaux, d'âge tardiglaciaire sensu lato, et la retombée supérieure de Rouzat, observée au sein des travertins de la source de Rouzat, à composition de trachybasalte, d'âge holocène ancien sensu lato (Vernet, 1992).

\section{3 - Conclusions}

La Limagne occidentale a donc été affectée directement par plusieurs épisodes éruptifs de la Chaîne des Puys au Tardiglaciaire et à l'Holocène et il donc aujourd'hui possible d'en proposer une téphrostratigraphie très fiable. 
Ces éruptions ont affecté les biotopes de façon beaucoup plus importante qu'on ne le pensait généralement. De larges saupoudrages trachyandésitiques sont intervenus pendant l'Alleröd, modifiant les environnements végétaux et perturbant les dispositifs d'occupation du territoire par les populations préhistoriques. Plus net encore est l'impact des éruptions trachyandésitiques et trachytiques au cours du Boréal : les téphras CF2, CF3 et CF4 se superposent sans paléosol intercalé et sont suivis par les recouvrements trachytiques fins du système Kilian-Vasset (CF5) et les divers produits distaux de l'éruption du Chopine, puis par les retombées du Pariou (CF6). Enfin, l'explosion d'un dôme primitif du Clierzou (CF7) est pénécontemporaine des premières installations du Néolithique ancien. Des téphras très récents (charnière Boréal/Atlantique et seconde moitié de l'Atlantique) ont été signalés sur le plateau des Dômes (Juvigné et al., 1986) mais restent à déceler en plaine. Ces apports téphriques répétés ont accéléré le comblement des dépressions de Limagne et perturbé considérablement l'évolution des marais.

Ces résultats obligent désormais les archéologues à reconsidérer l'impact du volcanisme de la Chaîne des Puys sur l'environnement régional, impact que l'on ne saurait plus minimiser a priori, d'autant que les études palynologiques confirment son importance. II reste à quantifier ces phénomènes et à évaluer précisément leurs conséquences pour les peuplements préhistoriques régionaux, travail collectif et de longue haleine.

Remerciements : ce travail a bénéficié du soutien des Régions Aquitaine et Auvergne au programme Espaces volcaniques préhistoriques conduit dans le cadre du GDR 1122 CNRS Hommes et volcans avant l'Histoire. Les auteurs ont une dette particulière envers Guy Camus et dédient ce travail à sa mémoire. 


\section{Références}

Baudry D. \& Camus G., 1972. Les projections volcaniques de la Chaîne des Puys et leurs utilisations. Bull. du B.R.G.M.,, 2), II, 2, 1-52.

Bentor Y.K., 1955. La chaine des Puys (Massif Central français) recherches géologiques et pétrographiques. Bull. du Service de la Carte Géologique de la France, №242, Tome LII, 1954, 787 p., fig. 75, tabl. CLXX.

Brousse R., Delibrias G., Labeyrie J., Rudel A., 1969. Eléments de chronologie des éruptions de la Chaîne des Puys. Bulletin de la Société géologique de France, $7^{\text {ème }}$ série, $\mathrm{XI}, 770-793$.

Camus G.,1975. La Chaîne des Puys : étude structurale et volcanologique. Annales Scientifiques de l'Université de Clermont, №56, Géol. Minéral., fasc. 28, fig. 68, 322 p.

Daugas J.P. et Tixier L., 1975. Variations paléoclimatiques de la Limagne d'Auvergne. Bulletin de l'Association française pour l'étude du Quaternaire, 47, 203-235.

Faïn J., Erramli H., Miallier D., Montret M., Sanzelle S., 1986. Datation par thermoluminescence d'un appareil volcanique trachytique : le Puy de Dôme. 11è Réunion des Sciences de la Terre, Clermont-Ferrand, S.G.F. Ed., Paris, 63.

Faïn J., Erramli H., Miallier D., Montret M., Sanzelle S., 1991. Datation par thermoluminescence d'un appareil volcanique trachytique : le Puy de Dôme. in J.P. Raynal et D. Miallier : Datation et Caractérisation des Milieux Pléistocènes, Cahiers du Quaternaire, CNRS Ed., n 16, 53-62.

Gourgaud A. et Camus G. (1984 ) - Magma Mixing at La Nugère Volcano (Chaîne des Puys, Massif Central, France) : Role in the Trachyandesites Genesis. Bull. Volcanol., 47/4, p. 781-805.

Etlicher B., Janssen C., Juvigné E., Van Leeuwen J.F.N. (1987) - Le Haut Forez (Massif Central, France) après le pléniglaciaire würmien : environnement et tephra du volcan de la Nugère. Bulletin de l'Association française pour l'étude du Quaternaire , 4, 229-239.

Fischer R.V., 1961. Proposed classification of volcaniclastic sediments and rocks. Geological Society of America Bulletin, 72, 1409-1414.

Guérin G., 1983. Thermoluminescence des plagioclases. Méthode de datation du volcanisme. Applications aux domaines volcaniques français : 
Chaîçuuuuuuuèèèèèène des Puys, Mont Dore et Cézalier, Bas Vivarais. Thèse d'état, Université Pierre et Marie Curie, Paris, 253 p.

Juvigné E., 1987. Un marqueur stratigraphique à large dispersion dans le Massif Central français : la retombée du volcan Chopine vieille d'environ 8500 ans B.P. C. R. Acad. Sc., Paris, t. 304, série II, $\mathrm{n}^{\circ} 4$, 187-190.

Juvigné E., 1991. Distribution de vastes retombées volcaniques originaires de l'Eifel et du Massif Central aux temps post-glaciaires dans le NE de la France et les régions voisines. C. R. Acad. Sc., Paris, tome 312, Série II, 415-420, 2 fig., 2 tabl.

Juvigné E., 1992. Distribution of widespread Late Glacial and Holocene tephra beds in the french Central Massif. Quaternary International, 13/14, 180-185.

Juvigné E., Bastin B., Gewelt M., 1986. Découverte de retombées volcaniques d'âge Holocène dans la Chaîne des Puys septentrionale (Massif Central, France). Revue des Sciences naturelles d'Auvergne, $\mathrm{n}^{\circ}$ 52, 33-36.

Juvigné E., Kroonenberg S.B., Weldkamp A., El Arabi A., Vernet G., 1992. Widespread Alleröd and Boreal trachyandesitic to trachytic tephra layers as stratigraphical markers in the Massif Central, France. Quaternaire, 3 (3-4), 137-146.

Le Bas, M.J., Le Maitre R.W., Streckeisen A., Zanettin B. (1986) - A chemical classification of volcanic rocks based on the total alkali-silica diagram. J. Petrology, 27, 745-750, Tulsa.

Michon L., 1996. Le Cratère Kilian et le Puy Vasset : mécanismes éruptifs et distinction des produits proximaux et distaux. Comparaison avec le cratère-lac Pavin et le Puy Chopine. Mémoire de travail d'étude et de recherche, Université de Clermont II, 20 p.

Raynal J.P., Paquereau M.M. et Daugas J.P., 1981. Contribution à l'étude chronostratigraphique des formations volcano-sédimentaires de la Chaîne des Puys. Nouv. Arch. Mus. Hist. nat. Lyon, Fasc. 19, 59-64, 2 fig.

Raynal J.P., Miallier D., Vernet G., Faïn J., Camus G., Montret M., Daugas J.P., Sanzelle S, 1989. Extension de la datation par thermoluminescence à une retombée trachy-andésitique rapportée au Puy de Pariou (Chaîne des Puys, France). C. R. Acad. Sc., Paris, t. 308, Série II, 1547-1552.

Raynal J.P., Vernet G., Vivent D., 1998. Des volcans et des hommes depuis le dernier interglaciaire en Basse Auvergne (Massif Central, France). in Albore- 
Livadie C. e Ortolani F. ed, Il systema uomo-ambiente tra passato e presente, Edipuglia, 197-220.

Vernet G., 1992. Message du volcanisme régional dans les formations quaternaires de Limagne occidentale (Massif central français). Minéraux denses et retombées. Thèse de l'Université de Bordeaux 1, $\mathrm{n}^{\circ} 724,335$ p.

Vernet G. et Paquereau M.M., 1986. Le cours moyen de la Morge (Puy-de-Dôme) au Pléistocène : éléments de chronologie et de paléoenvironnements. 11 ème Réunion des Sciences de la Terre, Clermont-Ferrand, S.G.F. Ed., 181.

Vernet G. et Paquereau M.M., 1991. Le cours moyen de la Morge et de ses affluents (Puy de Dôme, France) au Pléistocène : éléments de chronologie et de paléoenvironnements. in J.P. Raynal et D. Miallier : Datation et caractérisation des milieux pléistocènes, Cahiers du Quaternaire, $\mathrm{n}^{\circ}$ 16, CNRS. Ed., 431-449.

Vernet G. et Raynal J.P., 1995. La Téphra des Roches, marqueur du volcanisme contemporain de la fin du Magdalénien dans le Massif Central français. C. $R$. Acad. Sc., Paris , t. 321, Série lla, 713-720.

Vernet G. et Raynal J.P., Kieffer G., Camus G. ${ }^{+}$, Guadelli J.L., Vivent D., Miallier D., Sanzelle S., Pilleyre T., Fain J., Montret M., 2001 - L'éruption trachytique du Puy Chopine (Puy de Dôme, France) : impacts proximaux et distaux et implications écoarchéologiques (ce volume).

Vernet G., Raynal J.P., Miallier D., Pilleyre T., Faïn J., Sanzelle S., Montret M., 1990. La retombée de la Moutade, marqueur stratigraphique de l'Alleröd en Limagne septentrionale (Massif Central, France). C. R. Acad. Sc., Paris, t. 310, série II, 1077-1082.

Vernet G., Raynal J.P., Vivent D., 1996. La téphra de la rue Sous-les-Vignes, marqueur en Limagne du volcanisme trachytique postglaciaire de la Chaîne des Puys. C. R. Acad. Sc., Paris, t. 323, série lla, 325-331.

Vernet G., Raynal J.P., Faïn J., Miallier D., Montret M., Pilleyre T., Sanzelle S., 1998. Tephrostratigraphy of the last $160 \mathrm{ka}$ in Western Limagne (France). Quaternary International, vol. 47/48, 139-146. 